\title{
Short Summaries
}

\author{
Derek Richards \\ Centre for Evidence-based Dentistry, Oxford, UK
}

These short summaries are of a number of recently updated Cochrane Oral Health

Group reviews which have had no significant changes since their last publication.

Evidence-Based Dentistry (2008) 9, 91-92. doi:10.1038/sj.ebd.6400605

\section{Hyperbaric oxygen therapy and implant placement}

\begin{abstract}
Esposito M, Grusovin MG, Patel S, Worthington HV, Coulthard P.

Interventions for replacing missing teeth: hyperbaric oxygen therapy for irradiated patients who require dental implants. Cochrane Database Syst Rev 2008; issue 1

People who have undergone radiotherapy and those who have also undergone surgery for cancer in the head and neck region may particularly benefit from reconstruction with implants. Hyperbaric oxygen therapy (HBO) has been advocated as a way of improving the success of implant treatment in patients who have undergone radiotherapy, but this remains a controversial issue. This review only identified one randomised controlled trial (RCT) in which 13 patients received HBO therapy compared with 13 who did not. There was no statistically significant difference for prosthesis and implant failures, postoperative complications and patient satisfaction between the two groups. Therefore, it appears that $\mathrm{HBO}$ therapy in irradiated patients who require dental implants may not offer any appreciable clinical benefits and there is a need for more RCT to ascertain the effectiveness of $\mathrm{HBO}$ in this group of subjects.
\end{abstract}

\section{Maintaining soft tissue health around dental implants}

\section{Grusovin MG, Coulthard P, Jourabchian E, Worthington HV, Esposito MAB.}

Interventions for replacing missing teeth: maintaining and recovering soft tissue health around dental implants. Cochrane Database Syst Rev 2008; issue 1

Following the placement of dental implants, it is important to institute effective treatments to recover and or maintain soft tissue health around them. A number of different maintenance regimens have been suggested so this trial looked at which are the most effective.

Eighteen RCT were identified, nine eventually being included and involving a total of 238 patients. Followup ranged between 6 weeks and 1 year. As each RCT tested different interventions, no meta-analysis was conducted. Listerine (Pfizer Consumer Healthcare, Morris Plains, New Jersey, USA) mouthwash showed a reduction of $54 \%$ in plaque and $34 \%$ in marginal bleeding compared with a placebo. Two trials evaluated the efficacy of powered and sonic toothbrushes compared with manual toothbrushing and showed no statistically significant differences, although more patients liked the sonic brush. No statistical differences were found between brushing with a hyaluronic or a chlorhexidine gel, between cleaning with an etching gel or manually, between injecting a chlorhexidine or a physiological solution inside the implant, and between submucosal minocycline and a chlorhexidine gel. When an amine fluoride/stannous fluoride (AmF/ SnF2) mouthrinse was compared with a chlorhexidine one, no statistically significant differences were found for implant failures and staining index; patients preferred and had less taste change with the AmF/ SnF2 mouthrinse. Selfadministered subgingival chlorhexidine irrigation resulted in statistically significantly lower plaque and marginal bleeding than a chlorhexidine mouthwash, but the mouthwash was given at a suboptimal dosage.

Overall, the review concluded that there is no evidence from trials that powered or sonic toothbrushes are better than manual brushes and that brushing with a hyaluronic gel outdoes brushing with a chlorhexidine gel. Of the professionally administered treatments, there is no evidence that phosphoric acid is better than scaling and polishing, that chlorhexidine enclosed in the inner part of implants is superior to physiological solution or that a topical antibiotic inserted submucosally is any better than a chlorhexidine gel. There is some evidence, however, that Listerine antibacterial mouthrinse, used twice a day after brushing, can help to keep gums healthy.

\section{Dental recall frequency}

Beirne P, Clarkson JE, Worthington HV.

Recall intervals for oral health in primary care patients. Cochrane Database Syst Rev 2007; issue 4

The frequency with which patients should attend for a dental checkup and the potential effects on oral health of altering recall intervals between checkups have been the subject of ongoing international debate for almost 3 decades. Although recommendations regarding optimal recall intervals vary between countries and dental healthcare systems, 6-monthly dental checkups have traditionally been advocated by general dental practitioners in many developed countries.

The first version of this review was undertaken at the time that the UK National Institute for Health and Clinical Excellence (NICE) was developing guidance on dental recall (www.nice.org.uk/guidance/ CG19). The NICE guidance recommended that:

- The shortest interval between oral health reviews for all patients should be 3 months

- The longest interval between oral health reviews for patients younger than 18 years should be 12 months

- The longest interval between oral health reviews for patients aged 18 years and older should be 24 months

The updated review included only one study that was not included in the previous review so the review's original conclusions still stand: there is insufficient evidence from RCT to draw conclusions about potential beneficial and harmful effects of altering the recall interval between dental checkups. There is not enough evidence to support 
or refute the practice of encouraging patients to attend for dental checkups at 6-monthly intervals. High-quality RCT are needed to meet the outcomes listed in this review in order for the review to address its objectives.

\section{Routine scale and polish for periodontal health in adults}

\section{Beirne P, Worthington HV, Clarkson JE.}

Routine scale and polish for periodontal health in adults. Cochrane Database Syst Rev 2007; issue 4

This review looks at the effectiveness of routine scaling and polishing and was, like that above, originally undertaken at the time of the NICE Dental Recall Review and formed part of its deliberations. As with the original review, this update could only include nice studies, all of which had a high risk of bias.

Two split-mouth studies provided data for the comparison between scale and polish versus no scale and polish. One study involved people attending a recall programme following periodontal treatment: it found no statistically significant differences for plaque, gingivitis and attachment loss between experimental and control units at each timepoint during the 1-year trial. The other study involved adolescents in a developing country who had high existing levels of calculus and who had not received any dental treatment for at least 5 years. It reported statistically significant differences in calculus and gingivitis (bleeding) scores between treatment and control teeth at 6, 12 and 22 months (in favour of 'scale and polished teeth') following a single scale and polish provided at baseline to treatment teeth. For comparisons between routine scale and polish provided at different time intervals, there were some statistically significant differences in favour of scaling and polishing provided at more frequent intervals: 2 weeks versus 6 months, 2 weeks versus 12 months (for the outcomes plaque, gingivitis, pocket depth and attachment change); and 3 months versus 12 months (for the outcomes plaque, calculus and gingivitis). No studies compared the effects of scaling and polishing provided by dentists or professionals complementary to dentistry.

As with the previous review, the original conclusion stands: the research evidence is of insufficient quality to reach any conclusions regarding the beneficial and adverse effects of routine scaling and polishing for periodontal health or about the effects of providing this intervention at different time intervals. High-quality clinical trials are required to address the basic questions posed in this review. 